\title{
Assessment of knowledge and attitude of Rural Population about HIV/AIDS in Raigad District, India
}

\author{
${ }^{1}$ Sachin Solat, ${ }^{2}$ GD Velhal, ${ }^{3}$ Hemant Mahajan, ${ }^{4}$ Amrita Rao, ${ }^{5}$ Bhuwan Sharma \\ ${ }^{I}$ Assistant Professor, PDVVPF's Medical College, Ahmednagar, MUHS Nashik, India \\ ${ }^{2}$ Additional Professor, ${ }^{4}$ Assistant Professor, ${ }^{5}$ Junior Resident, TN Medical College, Mumbai, MUHS Nashik, \\ India \\ ${ }^{3}$ Assistant Professor, RCSM GMC Kolhapur, MUHS Nashik, India
}

\begin{abstract}
Objective: To assess the knowledge and attitude of rural population about HIV/AIDS in Raigad district. Material and Methods: A descriptive epidemiological study was conducted during January 2009 to April 2009 at Mahad Taluka of Raigad District, India. Total of 724 individuals were selected by using stratified random sampling. The information was gathered by personal interview using semi-structured proforma. Results: Total 724 individuals were interviewed with 202 (27.9\%) males and 522 (72.1\%) females. Overall awareness about symptoms of HIV/AIDS, its transmission routes and preventive measures was very poor. $383(52.9 \%)$ individual knew correct routes of transmission while only 289 (39.9\%) individuals knew how to prevent HIV infection transmission. 317 (43.8\%) individual never heard about condom and 117 (17.2\%) population knew correct purpose of condom. Composite scores of Knowledge and attitude were low (below 50\%) specifically in females, unmarried and illiterate individuals. Conclusion: Thus the knowledge of rural people about HIV / AIDS, its transmission and prevention should be improved with help of vigorous campaigning, training workshops, HIV / AIDS education sessions with special focus on migrant workers and their families.
\end{abstract}

Keywords: AIDS, Attitude, HIV, Knowledge, Rural

\section{Introduction}

When AIDS first emerged, no one could have predicted how the epidemic would spread across the world and how many millions of lives it would change. There was no real idea what caused it and consequently no real idea how to protect against it. Now we know from bitter experience that AIDS is caused by the HIV virus, and that it can devastate families, communities and whole continents. We have seen the epidemic knock decades off countries' national development, widen the gulf between rich and poor nations and push already stigmatized groups closer to the margins of society. We are living in an 'international' society, and HIV has become the first truly 'international' epidemic, easily crossing oceans and borders. It has been noted that a country with a very high HIV prevalence rate will often see this rate eventually stabilize, and even decline. It is disappointing that the global numbers of people infected with HIV continue to increase, despite the fact that effective prevention strategies already exist. Number of people living with HIV in 2008 was 33.4 million. Of these 33.4 million 2.7 million were newly infected patients in 2008 and AIDS related deaths were 2 million. ${ }^{(\mathbf{1})}$

The Government of India estimates that in 2007, about 2.31 million Indians were living with HIV (1.8 -2.9 million) with an adult prevalence of 0.34 percent. ${ }^{(2)}$ Demographically the second largest country in the world, India has also the third largest number of people living with HIV/AIDS. HIV estimates derived using globally comparable methods and findings from the independent Impact Assessment Study shows that the National AIDS Control Programme is progressing steadily towards the objective of halting and reversing the HIV epidemic in India over the period 2007- 2012. Available evidence on HIV prevalence and future statistical projections shows signs of stabilization of HIV epidemic in India at national level. Provisional estimates show that there are 22.7 lakh People living with HIV/AIDS in India by the end of 2008 with an estimated adult HIV prevalence of 0.29 percent. ${ }^{(3)}$

Maharashtra has the highest rate of urbanization i.e. $42 \%$, major national highways pass through the state, it has a high rate of migrant and floating population, and there exists a well established sex industry in the state and industrialization leading to availability of cash money in the hands of industrial workers. The bed occupancy in many hospitals due to HIV positive persons has been as high as $25 \%$ to $30 \%$, the ANC (Antenatal cases) HIV prevalence is around 1\% and the HIV prevalence in STD clinics is $10.4 \%$. There are 216748 registered HIV positive persons in the state today and an estimated figure of 8, 51,420 people living with HIV / AIDS. The total number of AIDS cases is 47,386 and those who have died are 2958. ${ }^{(4)}$

Maharashtra ranks first nationally in the proportion of total migrants. Maharashtra is India's leading industrial state and attracts male from almost all states of India. Census data suggest a predominance of interstate migration to districts of Thane, Mumbai suburban, Nashik and Pune. A considerable volume of inter-state 
in-migrants come from some of the high prevalence districts of Karnataka and Andhra Pradesh. When comparing Maharashtra to Andhra Pradesh (Verma et.al. 2007) and Karnataka (Saggurti et.al. 2008) the relationship between migration and HIV is strong. ${ }^{(5)}$

Thane, Pune, Nashik, Mumbai (urban), Mumbai (suburban) and Nagpur districts received more than half of male migrants during 1991-2001. These districts also have recorded a high HIV prevalence among pregnant women attending ANC clinics. In addition, the primary out-migration districts such as Sangli, Satara, Solapur, Jalgaon and Bid have recorded a high HIV prevalence. Thus, both the origin and destination districts demonstrate a link to HIV. ${ }^{(5)}$

These facts indicate the transmission of HIV from urban to rural areas and from high risk to low risk behaviour groups. If left unattended, this problem may spread like a fire and all the gains of the project activities so far, are likely to get major setback. Hence it is necessary at this stage to plan and operationalize the appropriate preventive and control measures against HIV/AIDS to control the transmission especially from the migrants.

\section{Materials And Methodology}

The present study was a community based descriptive epidemiological study carried out during January 2009 to April 2009 in Mahad Taluka in Raigad District of Konkan region of Western Maharashtra. District has sex ratio of 975 females per 1000 males and literacy rate of $60.4 \%$. Almost $41 \%$ of the population lies below poverty line.

For the purpose of present study, it was decided to include members from the important stakeholders as well as beneficiaries of PRIDE-India, who are also the influential members in the community, in the 40 villages covered by PRIDE-India. They are as follows:

\begin{tabular}{|l|c|}
\hline Self help groups members & 3000 \\
\hline Sponsored children & 1500 \\
\hline Adolescent boys & 400 \\
\hline Adolescent girls & 400 \\
\hline No. of families where at least1 member has migrated to Mumbai & 1000 \\
\hline Panchayat Raj Institutes members (Male) & 200 \\
\hline Anganwadi workers & 150 \\
\hline High school Teachers & 60 \\
\hline Preschool Teachers & 35 \\
\hline & 6745 \\
\hline
\end{tabular}

It was proposed to include at least, $10 \%$ i.e. 700 individuals from these groups for the purpose of survey for assessment of knowledge and attitude. Sampling technique was Stratified random sampling. Stratification was done according to the categories mentioned above. 724 individuals were selected by keeping Inclusion and exclusion criteria in mind.

After finalizing the study design, it was thoroughly discussed and administrative approval was taken from PRIDE-India officials at Mahad. Permission for the present study was taken from the Dean and Head of the Department of Preventive and Social Medicine TN Medical College, Mumbai. Ethics committee clearance for the present study was taken after submitting the study protocol.

Basic information about study area, study population and NGO activities was collected from PRIDEIndia NGO by studying previous records. Data collection tools were prepared: consent forms and preformed, pretested, semi-structured, open-ended questionnaire, all in the local language of communication i.e. Marathi.

\subsection{Knowledge}

Knowledge was assessed by using the composite scale giving scores to different attributes of knowledge regarding STIs and HIV/AIDS presented in interview schedule.

\begin{tabular}{r|r|rr|}
\hline Sr. & Questions & Answer / Score \\
\hline \multicolumn{3}{c|}{ www.iosrjournals.org } & $32 \mid$ Page
\end{tabular}


Assessment Of Knowledge And Attitude Of Rural Population About HIV/AIDS In Raigad

\begin{tabular}{|c|c|c|c|c|}
\hline No. & & Yes & \multicolumn{2}{|c|}{ No / Don't know } \\
\hline 1 & Heard of STIs? & 1 & \multicolumn{2}{|c|}{0} \\
\hline 2 & Are STIs curable? & 1 & \multicolumn{2}{|c|}{0} \\
\hline 3 & Have you heard of HIV/AIDS? & 1 & \multicolumn{2}{|c|}{0} \\
\hline 4 & $\begin{array}{l}\text { Is there any difference between HIV \& } \\
\text { AIDS? }\end{array}$ & 1 & \multicolumn{2}{|l|}{0} \\
\hline 5 & $\begin{array}{l}\text { Is there any relation between HIV/AIDS \& } \\
\text { STIs? }\end{array}$ & 1 & \multicolumn{2}{|l|}{0} \\
\hline 6 & Is HIV/AIDs completely curable? & 0 & \multicolumn{2}{|c|}{$\begin{array}{c}\text { No=1 / } \\
\text { Don't know }=0\end{array}$} \\
\hline 7 & Availability of HIV testing facilities? & 1 & \multicolumn{2}{|c|}{0} \\
\hline 8 & Availability of HIV treatment facilities? & 1 & \multicolumn{2}{|c|}{0} \\
\hline 9 & Desire more information on HIV? & 1 & \multicolumn{2}{|c|}{0} \\
\hline 10 & Have you heard of condom? & 1 & \multicolumn{2}{|l|}{0} \\
\hline 11 & Do you discuss health and sex issues? & 1 & \multicolumn{2}{|c|}{0} \\
\hline 12 & Necessity of HIV testing in pregnant women? & 1 & \multicolumn{2}{|c|}{0} \\
\hline 13 & Undergone HIV testing? & 1 & \multicolumn{2}{|c|}{0} \\
\hline & & Correct & Partially correct & $\begin{array}{c}\text { Wrong / } \\
\text { Don't know }\end{array}$ \\
\hline 14 & Meaning of STIs? & 2 & 1 & 0 \\
\hline 15 & Ways of transmission of STIs? & 2 & 1 & 0 \\
\hline 16 & Preventive measures for STIs? & 2 & 1 & 0 \\
\hline 17 & Symptoms of HIV? & 2 & 1 & 0 \\
\hline 18 & Symptoms of AIDS? & 2 & 1 & 0 \\
\hline 19 & Transmission of HIV/AIDS? & 2 & 1 & 0 \\
\hline 20 & Preventive measures for HIV/AIDS? & 2 & 1 & 0 \\
\hline 21 & Purpose of condom use? & 2 & 1 & 0 \\
\hline
\end{tabular}

Maximum obtainable score was 29.

Score of 0-13 considered as poor score; Scores of 14-29 considered as Good Score

\section{$2.2 \quad$ Attitude}

Attitude was assessed by using the composite scale giving scores to different attributes of attitude regarding STIs and HIV / AIDS presented in the interview schedule.

\begin{tabular}{|c|l|c|c|c|}
\hline $\begin{array}{c}\text { Sr. } \\
\text { No. }\end{array}$ & \multicolumn{1}{|c|}{ Statements } & Yes & No & Don't know \\
\hline 1 & $\begin{array}{l}\text { STIs can be cured by having sex relation with young } \\
\text { unmarried woman? }\end{array}$ & 0 & 1 & 0 \\
\hline 2 & $\begin{array}{l}\text { It is mandatory to subjects both the partners for HIV } \\
\text { testing before marriage? }\end{array}$ & 0 & 1 & 0 \\
\hline 3 & $\begin{array}{l}\text { It is dangerous to keep HIV infected individual at } \\
\text { home? }\end{array}$ & 0 & 1 & 0 \\
\hline 4 & $\begin{array}{l}\text { Complete elimination of prostitution is necessary to } \\
\text { prevent HIV transmission? }\end{array}$ & 0 & 1 & 0 \\
\hline 5 & HIV infected females should never get conceived? & 0 & 1 & 0 \\
\hline 6 & $\begin{array}{l}\text { Those who are confirmed HIV negative after testing, } \\
\text { have no risk of becoming HIV infected any time in } \\
\text { the life? }\end{array}$ & 0 & 1 & 0 \\
\hline 7 & $\begin{array}{l}\text { It is possible to identify HIV infected individual } \\
\text { easily? }\end{array}$ & 0 & 1 & 0 \\
\hline 8 & STIs \& HIV/AIDS are transmitted by only females? & 0 & 1 & 0 \\
\hline 9 & $\begin{array}{l}\text { It is necessary to test each individual for HIV } \\
\text { infection? }\end{array}$ & 0 & 1 & 0 \\
\hline 10 & $\begin{array}{l}\text { It is dangerous to provide health care to HIV } \\
\text { infected individual? }\end{array}$ & 0 & 1 & 0 \\
\hline
\end{tabular}

Maximum obtainable score was 10 .

Score of $0-5$ is considered as poor score and 6-10 is good score. 


\subsection{Planning Of Survey}

The survey was planned in first week of January 2009. With the help of PRIDE-India officials the study population of 40 villages was divided into 4 Zones, namely, Pachad zone, Vinhere zone, Jui zone, Mahad zone.

\subsubsection{Identification Of Surveyors And The Supervisors}

Total 40 surveyors were identified which were Community Organizers (COs) and Balsevikas. 20 pairs were formed, preferably one male and one female in each pair. 4 Assistant Zonal officers (AZOs) were identified, one for each of the four zones. Each AZO was supposed to supervise activity of 5 (or 6) pairs of the surveyors and help them in case of difficulties.

\subsubsection{Training Of Surveyors And Supervisors}

All the 40 surveyors (20 Community Organizers and 20 Balsevikas) and the 4 Supervisors i.e. Assistant Zonal Officers were given 1 day training about how to ask the questions for interview schedule. This was done to have uniformity in questionnaire filling and avoid subjective errors. Their queries about some questions were solved on the spot. They were trained how to develop rapport with the respondents and how to get answers to certain embarrassing questions.

\subsubsection{Allotment Of Survey Areas}

The 40 surveyors were divided into 20 pairs, preferably one male and one female in each pair. Each pair was allotted 2 villages in such a way that surveyors were from different villages of residence than the ones allotted to them. This was done to avoid any bias on the part of the surveyors. The surveyors were also intimated about the route and mode of transport for reaching their allotted villages. The surveyors were also informed to be in contact with the respective supervisors i.e. Assistant Zonal Officers (AZOs) in case of any difficulties

\subsubsection{Field Survey}

Field survey was carried out over a period of 2 days. 20 villages were covered on day one and remaining 20 on the second day. Each pair covered one village on each day and completed interviews of the allotted participants. The surveyors introduced themselves to the participants, developed rapport, took their consent and then interviewed the participants. Interviews were taken in local language i.e. Marathi. It was made sure that each question was properly understood by the participants

Proper privacy was provided to all the participants. Care was taken that female participants were interviewed by the female surveyor and male participant by the male surveyor. An attempt was made to interview the spouse of the migrant, wherever applicable.

High school teachers and preschool teachers were requested to fill the forms, instead of taking their interview. This was mainly because the teachers would not be very comfortable to get interviewed by the community organisers and it is also more likely that they would express in a better fashion if given an opportunity to respond independently. Only those who have not undergone any training on HIV/AIDS were included in this exercise.

The four supervisors i.e. Assistant Zonal Officers (AZOs) supervised the work in their respective zones. AZOs also helped the surveyors in case of any difficulties. It was made sure that all interviews in that village are completed on the same day and not kept pending. Efforts were made on the part of the surveyors as well as the supervisors to minimize the mistakes in filling up the questionnaires.

\subsubsection{Statistical Analysis}

The data was checked for completeness. The data was coded and entered in Microsoft Office Excel. The data was filtered, processed and edited. The data was thoroughly analysed using SPSS 15.0 and Graph Pad Prism v5.01 statistical analysis software. Appropriate tests like Chi-square were applied.

\section{Results}

TABLE 1 describes, total number of 724 individuals were interviewed with 202 (27.9\%) males and 522 (72.1\%) females. Most of them $615(84.94 \%)$ were in the age group of 15 - 40 years, Hindu by religion (710, $98.06 \%)$, backward class $(294,40.60 \%)$, married $(408,56.35 \%)$, educated up to secondary school level (480, $66.29 \%)$, occupied as unskilled labourer $(374 / 479,78.8 \%)$ and socio-economic class IV (458, 63.26\%). Also, $547(75.55 \%)$ of participants have also reported various forms of addictions among their family members mainly tobacco consumption $(504,92.13 \%)$ in various forms. Only $99(49.0 \%)$ males and $83(15.90 \%)$ females mentioned that they were the decision makers on their health problems, while, only $87(43.07 \%)$ males and 288 (55.17\%) females said that they discuss health and sexual issues with family members. TABLES 2,3 show that males, married population, individuals having higher education level, self employed individuals and individuals 
Assessment Of Knowledge And Attitude Of Rural Population About HIV/AIDS In Raigad

having higher per capita income had more awareness about the symptoms of HIV and AIDS. Similarly TABLES 4 and 5 show awareness about transmission of HIV and Prevention of HIV infection was significantly more in people having higher education, self employed people, people having more per capita income and married population. TABLE 6 reveals although 383(52.9\%) individual knew about various routes of transmission, only 289 (75.6\%) of these 383 individuals knew how to prevent HIV infection transmission. TABLE 7 shows, 317 (43.8\%) individual never heard about condom. Only 117 (17.2\%) population knew correct purpose of condom. Significant relationship was seen between correct purpose of condom and population having higher education, males, married population, high per capita income and employed population. TABLES $8,9,10$ show only $106(14.6 \%)$ individuals had good knowledge (Composite knowledge score $\geq 14$ ) about HIV/AIDS and 165 (22.8\%) individuals had right attitude (Composite score 6 to 10) towards HIV/AIDS. Good knowledge and right attitude was significantly more in males, married population, individuals with higher education, population with high per capita income.

TABLE 1: Demographic Profile of Study Population (n - 724)

\begin{tabular}{|l|l|l|}
\hline Socio-demographic Profile & & Frequency \\
\hline \multirow{5}{*}{ Age in years } & Male & $202(27.9 \%)$ \\
\cline { 2 - 3 } & Female & $522(72.1 \%)$ \\
\hline \multirow{5}{*}{ Education } & $<20$ & $258(35.6 \%)$ \\
\cline { 2 - 3 } & $20-29$ & $153(21.1 \%)$ \\
\cline { 2 - 3 } & $30-39$ & $204(28.2 \%)$ \\
\cline { 2 - 3 } & $40-49$ & $73(10.1 \%)$ \\
\cline { 2 - 3 } & 50 \& above & $36(5 \%)$ \\
\hline \multirow{5}{*}{ Occupation } & Illiterate & $41(5.7 \%)$ \\
\cline { 2 - 3 } & Primary & $112(15.5 \%)$ \\
\cline { 2 - 3 } & Secondary & $468(64.6 \%)$ \\
\cline { 2 - 3 } & Higher Secondary & $94(13 \%)$ \\
\cline { 2 - 3 } & Graduation and above & $9(1.2 \%)$ \\
\hline \multirow{5}{*}{ Religion } & Self employed & $29(4 \%)$ \\
\cline { 2 - 3 } & Public Private sector employee & $59(8.2 \%)$ \\
\cline { 2 - 3 } & Unskilled laborer & $374(51.7 \%)$ \\
\cline { 2 - 3 } & Unemployed & $262(36.6 \%)$ \\
\hline Caste & Hindi & $710(98.06 \%)$ \\
\cline { 2 - 3 } & Muslim & $7(0.97 \%)$ \\
\cline { 2 - 3 } & Others & $7(0.97 \%)$ \\
\hline Marital Status & Backward Class & $294(40.6 \%)$ \\
\cline { 2 - 3 } & Others & $430(59.4 \%)$ \\
\hline Per Capita income per month & Married & $408(56.4 \%)$ \\
\cline { 2 - 3 } & Unmarried & $298(41.2 \%)$ \\
\cline { 2 - 3 } & Divorced and Others & $18(2.4 \%)$ \\
\hline & Class I & $29(4.01 \%)$ \\
\cline { 2 - 3 } & Class II & $56(7.73 \%)$ \\
\cline { 2 - 3 } & Class III & $181(25 \%)$ \\
\cline { 2 - 3 } & Class IV & $458(63.26 \%)$ \\
\hline & & \\
\hline
\end{tabular}

TABLE 2: Ever heard of HIV/ AIDS (n-724)

\begin{tabular}{|c|c|c|c|c|}
\hline Category & & Yes & No & Chi-square test \\
\hline \multirow[t]{2}{*}{ Sex } & Male & $194(96.04 \%)$ & $8(3.96 \%)$ & \multirow{2}{*}{$\begin{array}{l}\mathrm{x}^{2}-21.9 ; \mathrm{p} \text {-value }<0.01 \\
\text { Significant Association }\end{array}$} \\
\hline & Female & $432(82.8 \%)$ & $90(17.2 \%)$ & \\
\hline \multirow{5}{*}{$\begin{array}{l}\text { Age Group in } \\
\text { years }\end{array}$} & $<20$ & $200(77.5 \%)$ & $58(22.5 \%)$ & \multirow{5}{*}{$\begin{array}{l}\mathrm{x}^{2}-36.7 ; \mathrm{p} \text { value }<0.01 \\
\text { Significant Association }\end{array}$} \\
\hline & $20-29$ & $140(91.5 \%)$ & $13(8.5 \%)$ & \\
\hline & $30-39$ & $195(95.6 \%)$ & $9(4.4 \%)$ & \\
\hline & $40-49$ & $62(84.9 \%)$ & $11(15.1 \%)$ & \\
\hline & $50 \&$ above & $29(80.6 \%)$ & $7(19.4 \%)$ & \\
\hline \multirow[t]{4}{*}{ Education } & Illiterate & $21(51.2 \%)$ & $20(48.8 \%)$ & \multirow{4}{*}{$\begin{array}{l}\mathrm{x}^{2}-55.9 ; \mathrm{p} \text { value }<0.01 \\
\text { Significant Association }\end{array}$} \\
\hline & Primary & $90(80.4 \%)$ & $22(19.6 \%)$ & \\
\hline & Secondary & $418(89.3 \%)$ & $50(10.7 \%)$ & \\
\hline & Higher Secondary & $88(93.6 \%)$ & $6(6.4 \%)$ & \\
\hline
\end{tabular}


Assessment Of Knowledge And Attitude Of Rural Population About HIV/AIDS In Raigad

\begin{tabular}{|c|c|c|c|c|}
\hline & Graduate \& above & $9(100 \%)$ & $0(0 \%)$ & \\
\hline \multirow[t]{4}{*}{ Occupation } & Self employed & $26(89.7 \%)$ & $3(10.3 \%)$ & \multirow{4}{*}{$\begin{array}{l}\mathrm{x}^{2}-15.8 ; \mathrm{p} \text { value }<0.01 \\
\text { Significant Association }\end{array}$} \\
\hline & $\begin{array}{l}\text { Public-private } \\
\text { sector employee }\end{array}$ & $54(91.5 \%)$ & $5(8.5 \%)$ & \\
\hline & Unskilled laborer & $337(90.1 \%)$ & $37(9.9 \%)$ & \\
\hline & Unemployed & $209(79.7 \%)$ & $53(20.2 \%)$ & \\
\hline \multirow[t]{3}{*}{ Religion } & Hindu & $622(87.8 \%)$ & $88(12.2 \%)$ & \multirow{3}{*}{$\begin{array}{l}\mathrm{x}^{2}-43.3 ; \mathrm{p} \text { value }<0.01 \\
\text { Significant Association }\end{array}$} \\
\hline & Muslim & $3(42.9 \%)$ & $4(57.1 \%)$ & \\
\hline & Others & $1(14.3 \%)$ & $6(85.7 \%)$ & \\
\hline \multirow[t]{2}{*}{ Caste } & Backward Class & $237(80.6 \%)$ & $57(19.4 \%)$ & \multirow{2}{*}{$\begin{array}{l}x^{2}-14.5 ; p \text { value }<0.01 \\
\text { Significant Association }\end{array}$} \\
\hline & Others & $389(90.5 \%)$ & $41(9.5 \%)$ & \\
\hline \multirow[t]{3}{*}{ Marital Status } & Married & $373(91.4 \%)$ & $35(8.6 \%)$ & \multirow{3}{*}{$\begin{array}{l}\mathrm{x}^{2}-19.8 ; \mathrm{p} \text { value }<0.01 \\
\text { Significant Association }\end{array}$} \\
\hline & Unmarried & $238(79.9 \%)$ & $60(20.1 \%)$ & \\
\hline & Divorced \& others & $15(83.3 \%)$ & $3(16.7 \%)$ & \\
\hline \multirow{4}{*}{$\begin{array}{l}\text { Per capita } \\
\text { Income per } \\
\text { month }\end{array}$} & Class I & $27(93.1 \%)$ & $2(6.9 \%)$ & \multirow{4}{*}{$\begin{array}{l}\mathrm{x}^{2}-7.7 ; \mathrm{p} \text { value }-0.052 ; \\
\text { Non-Significant } \\
\text { Association }\end{array}$} \\
\hline & Class II & $52(92.9 \%)$ & $4(7.1 \%)$ & \\
\hline & Class III & $163(90.1)$ & $18(9.9 \%)$ & \\
\hline & Class IV & $384(83.8 \%)$ & $74(16.2 \%)$ & \\
\hline
\end{tabular}

TABLE 3: Awareness about the symptoms of HIV/AIDS (n- 724)

\begin{tabular}{|c|c|c|c|c|c|c|}
\hline Category & & Yes & No & Wrong & Don't know & Chi-square test \\
\hline \multirow[b]{2}{*}{ Sex } & Male & $2(1 \%)$ & $64(31.7 \%)$ & $36(17.8 \%)$ & $100(49.5 \%)$ & \multirow{2}{*}{$\begin{array}{l}\mathrm{x}^{2}-2.1 ; \mathrm{p} \text {-value } \\
<0.054 \text {; Non- } \\
\text { Significant } \\
\text { Association }\end{array}$} \\
\hline & Female & $12(2.3 \%)$ & $156(29.9 \%)$ & $81(15.5 \%)$ & $273(52.3 \%)$ & \\
\hline \multirow{5}{*}{$\begin{array}{l}\text { Age } \\
\text { Groups in } \\
\text { years }\end{array}$} & $<20$ & $1(0.4 \%)$ & $58(22.5 \%)$ & $53(20.5 \%)$ & $146(56.6 \%)$ & \multirow{5}{*}{$\begin{array}{l}\mathrm{x}^{2}-29.6 ; \mathrm{p}- \\
\text { value }<0.01 \\
\text { Significant } \\
\text { Association }\end{array}$} \\
\hline & $20-29$ & $2(1.3 \%)$ & $45(29.4 \%)$ & $23(15 \%)$ & $83(54.3 \%)$ & \\
\hline & $30-39$ & $6(2.9 \%)$ & $77(37.8 \%)$ & $28(13.7 \%)$ & $93(45.6 \%)$ & \\
\hline & $40-49$ & $4(5.5 \%)$ & $29(39.7 \%)$ & $8(11 \%)$ & $32(43.8 \%)$ & \\
\hline & $50 \&$ above & $1(2.8 \%)$ & $11(30.6 \%)$ & $5(13.9 \%)$ & $19(52.8 \%)$ & \\
\hline \multirow{5}{*}{ Education } & Illiterate & $0(0 \%)$ & $8(19.5 \%)$ & $9(22 \%)$ & $24(58.5 \%)$ & \multirow{5}{*}{$\begin{array}{l}\mathrm{x}^{2}-32.9 ; \mathrm{p} \\
\text { value }<0.01 ; \\
\text { Significant } \\
\text { Association }\end{array}$} \\
\hline & Primary & $1(0.9 \%)$ & $30(26.8 \%)$ & $21(18.8 \%)$ & $60(53.6 \%)$ & \\
\hline & Secondary & $8(1.7 \%)$ & $142(30.3 \%)$ & $72(15.4 \%)$ & $246(52.6 \%)$ & \\
\hline & $\begin{array}{l}\text { Higher } \\
\text { Secondary }\end{array}$ & $3(3.2 \%)$ & $35(37.2 \%)$ & $14(14.9 \%)$ & $42(44.7 \%)$ & \\
\hline & $\begin{array}{l}\text { Graduate \& } \\
\text { above }\end{array}$ & $2(22.2 \%)$ & $5(55.6 \%)$ & $1(11.1 \%)$ & $1(11.1 \%)$ & \\
\hline \multirow{4}{*}{ Occupation } & Self employed & $2(6.9 \%)$ & $22(75.9 \%)$ & $2(6.9 \%)$ & $3(10.3 \%)$ & \multirow{4}{*}{$\begin{array}{l}\mathrm{x}^{2}-59.5 ; \mathrm{p}- \\
\text { value }<0.01 \\
\text { Significant } \\
\text { Association }\end{array}$} \\
\hline & $\begin{array}{l}\text { Public-private } \\
\text { sector employee }\end{array}$ & $2(3.3 \%)$ & $27(45.8 \%)$ & $7(11.9 \%)$ & $23(39 \%)$ & \\
\hline & Unskilled laborer & $8(2.1 \%)$ & $119(31.8 \%)$ & $58(15.5 \%)$ & $189(50.5 \%)$ & \\
\hline & Unemployed & $2(0.8 \%)$ & $52(19.9 \%)$ & $50(19.1 \%)$ & $158(60.3 \%)$ & \\
\hline \multirow{3}{*}{ Religion } & Hindu & $14(2 \%)$ & $217(30.6 \%)$ & $115(16.2 \%)$ & $364(51.3 \%)$ & \multirow{3}{*}{$\begin{array}{l}\mathrm{x}^{2}-1.5 ; \mathrm{p} \\
\text { value- } 0.8 ; \\
\text { Nonsignificant } \\
\text { Association }\end{array}$} \\
\hline & Muslim & $0(0 \%)$ & $1(14.3 \%)$ & $1(14.3)$ & $5(71.4 \%)$ & \\
\hline & Others & $0(0 \%)$ & $2(28.6 \%)$ & $1(14.3 \%)$ & $4(57.1 \%)$ & \\
\hline \multirow[b]{2}{*}{ Caste } & Backward Class & $3(1 \%)$ & $77(26.2 \%)$ & $50(17 \%)$ & $164(55.8 \%)$ & \multirow{2}{*}{$\begin{array}{l}\mathrm{x}^{2}-16 ; \mathrm{p} \text { value } \\
<0.01 ; \\
\text { Significant } \\
\text { Association }\end{array}$} \\
\hline & Others & $11(2.6 \%)$ & $143(33.3 \%)$ & $67(15.6 \%)$ & $209(48.5 \%)$ & \\
\hline \multirow{3}{*}{$\begin{array}{l}\text { Marital } \\
\text { Status }\end{array}$} & Married & $12(2.9 \%)$ & $160(39.2 \%)$ & $54(13.2 \%)$ & $182(44.6 \%)$ & \multirow{3}{*}{$\begin{array}{l}\mathrm{x}^{2}-43.2 ; \mathrm{p}- \\
\text { value }<0.01 \\
\text { Significant } \\
\text { Association }\end{array}$} \\
\hline & Unmarried & $2(0.7 \%)$ & $55(18.5 \%)$ & $60(20.1 \%)$ & $181(60.7 \%)$ & \\
\hline & $\begin{array}{l}\text { Divorced \& } \\
\text { others }\end{array}$ & $0(0 \%)$ & $5(27.8 \%)$ & $3(16.7 \%)$ & $10(55.5 \%)$ & \\
\hline
\end{tabular}


Assessment Of Knowledge And Attitude Of Rural Population About HIV/AIDS In Raigad

\begin{tabular}{|l|l|l|l|l|l|l|}
\hline \multirow{2}{*}{$\begin{array}{l}\text { Per capita } \\
\text { Income per } \\
\text { month }\end{array}$} & Class I & $3(10.3 \%)$ & $17(58.6 \%)$ & $2(6.9 \%)$ & $7(24.1 \%)$ & $\mathrm{x}^{2}-46.9 ; \mathrm{p}$ \\
\cline { 2 - 6 } & Class II & $4(7.1 \%)$ & $25(44.6 \%)$ & $5(8.9 \%)$ & $22(39.3 \%)$ & value $<0.01 ;$ \\
\cline { 2 - 6 } & Class III & $4(2.2 \%)$ & $56(30.9 \%)$ & $29(16 \%)$ & $92(50.8 \%)$ & Significant \\
\cline { 2 - 6 } & Class IV & $3(0.7 \%)$ & $122(26.6 \%)$ & $81(17.7 \%)$ & $252(55 \%)$ & Association \\
\hline
\end{tabular}

TABLE 4: Awareness about transmission of HIV/AID (n-724)

\begin{tabular}{|c|c|c|c|c|c|c|}
\hline Category & & Correct & $\begin{array}{l}\text { Partially } \\
\text { correct }\end{array}$ & Wrong & Don't know & Chi-square test \\
\hline \multirow[b]{2}{*}{ Sex } & Male & $22(10.9 \%)$ & $84(41.6 \%)$ & $32(15.8)$ & $64(31.7 \%)$ & \multirow{2}{*}{$\begin{array}{l}\mathrm{x}^{2}-3.1 ; \mathrm{p}-0.4 ; \\
\text { Non-Significant } \\
\text { Association }\end{array}$} \\
\hline & Female & $38(7.3 \%)$ & $239(45.8 \%)$ & $75(14.4 \%)$ & $170(32.6 \%)$ & \\
\hline \multirow{5}{*}{$\begin{array}{l}\text { Age } \\
\text { Groups }\end{array}$} & $<20$ years & $11(4.3 \%)$ & $92(35.7 \%)$ & $51(19.8 \%)$ & $104(40.3 \%)$ & \multirow{5}{*}{$\begin{array}{l}\mathrm{x}^{2}-48.8 ; \mathrm{p} \text {-value } \\
<0.01 ; \\
\text { Significant } \\
\text { Association }\end{array}$} \\
\hline & $20-29$ years & $11(7.2 \%)$ & $65(42.5 \%)$ & $20(13.1 \%)$ & $57(37.2 \%)$ & \\
\hline & $30-39$ years & $23(11.3 \%)$ & $109(53.4 \%)$ & $25(12.3 \%)$ & $47(23 \%)$ & \\
\hline & $40-49$ years & $11(15.1 \%)$ & $43(58.9 \%)$ & $7(9.6 \%)$ & $12(16.4 \%)$ & \\
\hline & $50 \&$ above & $4(11.1 \%)$ & $14(38.9 \%)$ & $4(11.1 \%)$ & $14(38.9 \%)$ & \\
\hline \multirow{5}{*}{ Education } & Illiterate & $1(2.4 \%)$ & $9(22 \%)$ & $12(29.3 \%)$ & $19(46.3 \%)$ & \multirow{5}{*}{$\begin{array}{l}\mathrm{x}^{2}-33.9 ; \mathrm{p} \text { value } \\
<0.01 ; \\
\text { Significant } \\
\text { Association }\end{array}$} \\
\hline & Primary & $7(6.3 \%)$ & $44(39.3 \%)$ & $20(17.9 \%)$ & $41(36.6 \%)$ & \\
\hline & Secondary & $37(7.9 \%)$ & $217(46.3 \%)$ & $63(13.5 \%)$ & $151(32.3 \%)$ & \\
\hline & $\begin{array}{l}\text { Higher } \\
\text { Secondary }\end{array}$ & $12(12.8 \%)$ & $48(51.1 \%)$ & $11(11.7 \%)$ & $23(24.4 \%)$ & \\
\hline & $\begin{array}{l}\text { Graduate \& } \\
\text { above }\end{array}$ & $3(33.3 \%)$ & $5(55.6 \%)$ & $1(11.1 \%)$ & $0(0 \%)$ & \\
\hline \multirow{4}{*}{ Occupation } & Self employed & $5(17.2 \%)$ & $17(58.6 \%)$ & $2(6.9 \%)$ & $5(17.2 \%)$ & \multirow{4}{*}{$\begin{array}{l}\mathrm{x}^{2}-28.3 ; \mathrm{p} \text {-value } \\
<0.01 ; \\
\text { Significant } \\
\text { Association }\end{array}$} \\
\hline & $\begin{array}{l}\text { Public-private } \\
\text { sector employee }\end{array}$ & $7(11.9 \%)$ & $32(54.2 \%)$ & $7(11.9 \%)$ & $13(22 \%)$ & \\
\hline & $\begin{array}{l}\text { Unskilled } \\
\text { laborer }\end{array}$ & $36(9.6 \%)$ & $175(46.8 \%)$ & $55(14.7 \%)$ & $108(28.9 \%)$ & \\
\hline & Unemployed & $12(4.6 \%)$ & $99(37.8 \%)$ & $43(16.4 \%)$ & $108(41.2 \%)$ & \\
\hline \multirow{3}{*}{ Religion } & Hindu & $59(8.3 \%)$ & $318(44.8 \%)$ & $104(14.6 \%)$ & $229(32.3 \%)$ & \multirow{3}{*}{$\begin{array}{l}\mathrm{x}^{2}-11.8 ; \mathrm{p} \\
\text { value<0.01; } \\
\text { Significant } \\
\text { Association }\end{array}$} \\
\hline & Muslim & $0(0 \%)$ & $2(28.6 \%)$ & $2(28.5 \%)$ & $3(42.9 \%)$ & \\
\hline & Others & $1(14.3 \%)$ & $3(42.9 \%)$ & $1(14.3 \%)$ & $2(28.5 \%)$ & \\
\hline \multirow[b]{2}{*}{ Caste } & Backward Class & $22(7.5 \%)$ & $111(37.8 \%)$ & $51(17.4 \%)$ & $110(37.4 \%)$ & \multirow{2}{*}{$\begin{array}{l}\mathrm{x}^{2}-11.8 ; \mathrm{p} \text { value } \\
<0.01 ; \\
\text { Significant } \\
\text { Association }\end{array}$} \\
\hline & Others & $38(8.8 \%)$ & $212(49.3 \%)$ & $56(13 \%)$ & $124(28.8 \%)$ & \\
\hline \multirow{3}{*}{$\begin{array}{l}\text { Marital } \\
\text { Status }\end{array}$} & Married & $45(11 \%)$ & $260(63.7 \%)$ & $33(8.1 \%)$ & $70(17.2 \%)$ & \multirow{3}{*}{$\begin{array}{l}x^{2}-185 ; p \text {-value } \\
<0.01 ; \\
\text { Significant } \\
\text { Association }\end{array}$} \\
\hline & Unmarried & $14(4.7 \%)$ & $55(18.5 \%)$ & $71(23.8 \%)$ & $158(53 \%)$ & \\
\hline & $\begin{array}{l}\text { Divorced \& } \\
\text { others }\end{array}$ & $1(5.6 \%)$ & $8(44.4 \%)$ & $3(16.7 \%)$ & $6(33.3 \%)$ & \\
\hline \multirow{4}{*}{$\begin{array}{l}\text { Per capita } \\
\text { Income per } \\
\text { month }\end{array}$} & Class I & $6(20.7 \%)$ & $18(62.1 \%)$ & $2(6.9 \%)$ & $3(10.3 \%)$ & \multirow{4}{*}{$\begin{array}{l}\mathrm{x}^{2}-40.5 ; \mathrm{p} \text { value } \\
<0.01 ; \\
\text { Significant } \\
\text { Association }\end{array}$} \\
\hline & Class II & $9(16.1 \%)$ & $33(58.9 \%)$ & $6(10.7 \%)$ & $8(14.3 \%)$ & \\
\hline & Class III & $18(9.9 \%)$ & $89(49.2 \%)$ & $27(14.9 \%)$ & $47(26 \%)$ & \\
\hline & Class IV & $27(5.9 \%)$ & $183(40 \%)$ & $72(15.7 \%)$ & $176(38.4 \%)$ & \\
\hline
\end{tabular}

TABLE 5: Awareness about prevention of HIV Infection (n-724)

\begin{tabular}{|l|l|l|l|l|l|l|}
\hline \multirow{2}{*}{ Category } & & Correct & $\begin{array}{l}\text { Partially } \\
\text { correct }\end{array}$ & Wrong & Don't know & Chi-square test \\
\hline \multirow{3}{*}{ Sex } & Male & $11(5.4 \%)$ & $84(41.6 \%)$ & $47(23.3 \%)$ & $60(29.7 \%)$ & $\mathrm{x}^{2}-6.5 ; \mathrm{p}-0.09 ;$ \\
& Female & $19(3.6 \%)$ & $175(33.5 \%)$ & $155(29.7 \%)$ & $173(33.1 \%)$ & $\begin{array}{l}\text { Non-Significant } \\
\text { Association }\end{array}$ \\
\hline \multirow{4}{*}{$\begin{array}{l}\text { Age } \\
\text { Groups } \\
\text { In years }\end{array}$} & $20-29$ & $2(0.8 \%)$ & $55(21.3 \%)$ & $91(35.3 \%)$ & $110(42.6 \%)$ & $\mathrm{x}^{2}-77 ; \mathrm{p}$-value \\
\cline { 2 - 6 } & $30-39$ & $4(2.6 \%)$ & $55(35.9 \%)$ & $47(30.7 \%)$ & $47(30.7 \%)$ & $<0.01 ;$ \\
\cline { 2 - 6 } & $40-49$ & $15(7.4 \%)$ & $98(48 \%)$ & $47(23 \%)$ & $44(21.6 \%)$ & $\begin{array}{l}\text { Significant } \\
\text { Association }\end{array}$ \\
\hline
\end{tabular}


Assessment Of Knowledge And Attitude Of Rural Population About HIV/AIDS In Raigad

\begin{tabular}{|c|c|c|c|c|c|c|}
\hline & $50 \&$ above & $2(5.5 \%)$ & $14(38.9 \%)$ & $6(16.6 \%)$ & $14(38.9 \%)$ & \\
\hline \multirow{5}{*}{ Education } & Illiterate & $0(0 \%)$ & $6(14.6 \%)$ & $15(36.6 \%)$ & $20(48.8 \%)$ & \multirow{5}{*}{$\begin{array}{l}\mathrm{x}^{2}-87.8 ; \mathrm{p} \text { value } \\
<0.01 ; \\
\text { Significant } \\
\text { Association }\end{array}$} \\
\hline & Primary & $1(0.9 \%)$ & $22(19.6 \%)$ & $37(33 \%)$ & $52(46.4 \%)$ & \\
\hline & Secondary & $15(3.2 \%)$ & $187(39.9 \%)$ & $127(27.1 \%)$ & $139(29.7 \%)$ & \\
\hline & $\begin{array}{l}\text { Higher } \\
\text { Secondary }\end{array}$ & $10(10.6 \%)$ & $40(42.5 \%)$ & $22(23.4 \%)$ & $22(23.4 \%)$ & \\
\hline & $\begin{array}{l}\text { Graduate \& } \\
\text { above }\end{array}$ & $4(44.4 \%)$ & $4(44.4 \%)$ & $1(11.1 \%)$ & $0(0 \%)$ & \\
\hline \multirow{4}{*}{ Occupation } & Self employed & $5(17.2 \%)$ & $14(48.3 \%)$ & $4(13.8 \%)$ & $6(20.7 \%)$ & \multirow{4}{*}{$\begin{array}{l}\mathrm{x}^{2}-32.4 ; \mathrm{p}- \\
\text { value }<0.01 \\
\text { Significant } \\
\text { Association }\end{array}$} \\
\hline & $\begin{array}{l}\text { Public-private } \\
\text { sector employee }\end{array}$ & $6(10.2 \%)$ & $25(42.4 \%)$ & $9(15.3 \%)$ & $19(32.2 \%)$ & \\
\hline & $\begin{array}{l}\text { Unskilled } \\
\text { laborer }\end{array}$ & $15(4 \%)$ & $133(35.6 \%)$ & $105(28.1)$ & $121(32.3 \%)$ & \\
\hline & Unemployed & $4(1.5 \%)$ & $87(33.2 \%)$ & $84(32.1 \%)$ & $87(33.2 \%)$ & \\
\hline \multirow{3}{*}{ Religion } & Hindu & $29(4.1 \%)$ & $256(36.1 \%)$ & $197(27.7 \%)$ & $228(32.1 \%)$ & \multirow{3}{*}{$\begin{array}{l}\mathrm{x}^{2}-3.9 ; \mathrm{p} \text { value } \\
<0.4 ; \text { Non- } \\
\text { Significant } \\
\text { Association }\end{array}$} \\
\hline & Muslim & $0(0 \%)$ & $1(14.3 \%)$ & $3(42.9 \%)$ & $3(42.8 \%)$ & \\
\hline & Others & $1(14.3 \%)$ & $2(28.6 \%)$ & $2(28.6 \%)$ & $2(28.6 \%)$ & \\
\hline \multirow[b]{2}{*}{ Caste } & Backward Class & $11(3.7 \%)$ & $99(33.7 \%)$ & $89(30.3 \%)$ & $95(32.3 \%)$ & \multirow{2}{*}{$\begin{array}{l}\mathrm{x}^{2}-18 ; \mathrm{p} \text { value } \\
<0.61 ; \text { Non- } \\
\text { Significant } \\
\text { Association }\end{array}$} \\
\hline & Others & $19(4.4 \%)$ & $160(37.2 \%)$ & $113(26.3 \%)$ & $138(32.1 \%)$ & \\
\hline \multirow{3}{*}{$\begin{array}{l}\text { Marital } \\
\text { Status }\end{array}$} & Married & $20(4.9 \%)$ & $204(40.2 \%)$ & $83(25.2 \%)$ & $101(29.7 \%)$ & \multirow{3}{*}{$\begin{array}{l}\mathrm{x}^{2}-92 ; \mathrm{p} \text {-value } \\
<0.01 ; \\
\text { Significant } \\
\text { Association }\end{array}$} \\
\hline & Unmarried & $9(3 \%)$ & $49(29.9 \%)$ & $114(31.5 \%)$ & $126(35.6 \%)$ & \\
\hline & $\begin{array}{l}\text { Divorced \& } \\
\text { others }\end{array}$ & $1(5.6 \%)$ & $6(33.3 \%)$ & $5(27.8 \%)$ & $6(33.3 \%)$ & \\
\hline \multirow{4}{*}{$\begin{array}{l}\text { Per capita } \\
\text { Income per } \\
\text { month }\end{array}$} & Class I & $8(27.6 \%)$ & $16(55.2 \%)$ & $2(6.9 \%)$ & $3(10.3 \%)$ & \multirow{4}{*}{$\begin{array}{l}\mathrm{x}^{2}-85.8 ; \mathrm{p} \\
\text { value }<0.01 \\
\text { Significant } \\
\text { Association }\end{array}$} \\
\hline & Class II & $9(16.1 \%)$ & $25(44.6 \%)$ & $10(17.8 \%)$ & $12(21.4 \%)$ & \\
\hline & Class III & $6(3.3 \%)$ & $66(36.5 \%)$ & $49(27.1 \%)$ & $60(33.1 \%)$ & \\
\hline & Class IV & $7(1.5 \%)$ & $152(33.2 \%)$ & $141(30.8 \%)$ & $158(34.5 \%)$ & \\
\hline
\end{tabular}

TABLE 6: Association between awareness of transmission and awareness of prevention (n-383)

\begin{tabular}{|c|c|c|c|c|c|c|}
\hline Category & & Correct & $\begin{array}{l}\text { Partially } \\
\text { correct }\end{array}$ & Wrong & Don't know & Chi-square test \\
\hline \multirow[b]{2}{*}{ Sex } & Male & $11(10.4 \%)$ & $84(79.2 \%)$ & $4(3.8 \%)$ & $7(6.7 \%)$ & \multirow{2}{*}{$\begin{array}{l}\mathrm{x}^{2}-16.1 ; \mathrm{p}< \\
0.01 ; \\
\text { Significant } \\
\text { Association }\end{array}$} \\
\hline & Female & $19(6.9 \%)$ & $175(63.2 \%)$ & $32(11.6 \%)$ & $51(18.4 \%)$ & \\
\hline \multirow{5}{*}{$\begin{array}{l}\text { Age } \\
\text { Groups } \\
\text { In years }\end{array}$} & $<20$ & $2(19 \%)$ & $55(53.4 \%)$ & $20(19.4 \%)$ & $26(25.2 \%)$ & \multirow{5}{*}{$\begin{array}{l}\mathrm{x}^{2}-40.1 ; \mathrm{p}- \\
\text { value }<0.01 \\
\text { Significant } \\
\text { Association }\end{array}$} \\
\hline & $20-29$ & $4(5.3 \%)$ & $55(72.4 \%)$ & $6(7.9 \%)$ & $11(14.5 \%)$ & \\
\hline & $30-39$ & $15(11.4 \%)$ & $98(74.2 \%)$ & $6(4.6 \%)$ & $13(9.8 \%)$ & \\
\hline & $40-49$ & $7(13 \%)$ & $37(68.5 \%)$ & $3(5.6 \%)$ & $7(12.9 \%)$ & \\
\hline & $50 \&$ above & $2(11.1 \%)$ & $14(77.8 \%)$ & $1(5.6 \%)$ & $1(5.6 \%)$ & \\
\hline \multirow{5}{*}{ Education } & Illiterate & $0(0 \%)$ & $6(60 \%)$ & $1(10 \%)$ & $3(30 \%)$ & \multirow{5}{*}{$\begin{array}{l}\mathrm{x}^{2}-63 ; \mathrm{p} \text { value } \\
<0.01 ; \\
\text { Significant } \\
\text { Association }\end{array}$} \\
\hline & Primary & $1(2 \%)$ & $22(43.1 \%)$ & $12(23.5 \%)$ & $16(31.4 \%)$ & \\
\hline & Secondary & $15(5.9 \%)$ & $187(73.6 \%)$ & $17(6.7 \%)$ & $35(13.8 \%)$ & \\
\hline & $\begin{array}{l}\text { Higher } \\
\text { Secondary }\end{array}$ & $10(16.7 \%)$ & $40(66.7 \%)$ & $6(10 \%)$ & $4(6.7 \%)$ & \\
\hline & $\begin{array}{l}\text { Graduate \& } \\
\text { above }\end{array}$ & $4(50 \%)$ & $4(50 \%)$ & $0(0 \%)$ & $0(0 \%)$ & \\
\hline \multirow{4}{*}{ Occupation } & Self employed & $5(22.7 \%)$ & $14(63.6 \%)$ & $2(9.1 \%)$ & $1(4.6 \%)$ & \multirow{4}{*}{$\begin{array}{l}\mathrm{x}^{2}-21.2 ; \mathrm{p}- \\
\text { value }<0.01 \\
\text { Significant } \\
\text { Association }\end{array}$} \\
\hline & $\begin{array}{l}\text { Public-private } \\
\text { sector employee }\end{array}$ & $6(15.4 \%)$ & $25(64.1 \%)$ & $3(7.7 \%)$ & $5(12.8 \%)$ & \\
\hline & Unskilled laborer & $15(7.1 \%)$ & $133(63 \%)$ & $25(11.9 \%)$ & $38(18 \%)$ & \\
\hline & Unemployed & $4(3.6 \%)$ & $87(78.4 \%)$ & $6(5.4 \%)$ & $14(12.6 \%)$ & \\
\hline
\end{tabular}


Assessment Of Knowledge And Attitude Of Rural Population About HIV/AIDS In Raigad

\begin{tabular}{|c|c|c|c|c|c|c|}
\hline \multirow[b]{3}{*}{ Religion } & Hindu & $29(7.7 \%)$ & $256(67.9 \%)$ & $35(9.3 \%)$ & $57(15.1 \%)$ & \multirow{3}{*}{$\begin{array}{l}\mathrm{x}^{2}-5.4 ; \mathrm{p} \\
\text { value }<0.25 \\
\text { Non- } \\
\text { Significant } \\
\text { Association }\end{array}$} \\
\hline & Muslim & $0(0 \%)$ & $1(50 \%)$ & $0(0 \%)$ & $1(50.0 \%)$ & \\
\hline & Others & $1(25 \%)$ & $2(50 \%)$ & $1(25 \%)$ & $0(0 \%)$ & \\
\hline \multirow[b]{2}{*}{ Caste } & Backward Class & $11(8.3 \%)$ & $99(74.4 \%)$ & $10(7.5 \%)$ & $13(9.8 \%)$ & \multirow{2}{*}{$\begin{array}{l}\mathrm{x}^{2}-6.1 ; \mathrm{p} \\
\text { value }<0.11 ; \\
\text { Non- } \\
\text { Significant } \\
\text { Association }\end{array}$} \\
\hline & Others & $19(7.6 \%)$ & $160(64 \%)$ & $26(10.4 \%)$ & $45(18 \%)$ & \\
\hline \multirow{3}{*}{$\begin{array}{l}\text { Marital } \\
\text { Status }\end{array}$} & Married & $20(6.6 \%)$ & $204(66.9 \%)$ & $32(10.5 \%)$ & $49(16 \%)$ & \multirow{3}{*}{$\begin{array}{l}\mathrm{x}^{2}-6.4 ; \mathrm{p}- \\
\text { value }<0.4 \\
\text { Non- } \\
\text { Significant } \\
\text { Association }\end{array}$} \\
\hline & Unmarried & $9(13 \%)$ & $49(71 \%)$ & $3(4.4 \%)$ & $8(11.5 \%)$ & \\
\hline & $\begin{array}{l}\text { Divorced \& } \\
\text { others }\end{array}$ & $1(11.1 \%)$ & $6(66.7 \%)$ & $1(11.1 \%)$ & $1(11.1 \%)$ & \\
\hline \multirow{4}{*}{$\begin{array}{l}\text { Per capita } \\
\text { Income per } \\
\text { month }\end{array}$} & Class I & $8(33.3 \%)$ & $16(66.7 \%)$ & $0(0 \%)$ & $0(0 \%)$ & \multirow{4}{*}{$\begin{array}{l}\mathrm{x}^{2}-48.7 ; \mathrm{p} \\
\text { value }<0.01 \\
\text { Significant } \\
\text { Association }\end{array}$} \\
\hline & Class II & $9(21.4 \%)$ & $25(59.5 \%)$ & $5(11.9 \%)$ & $3(7.1 \%)$ & \\
\hline & Class III & $6(5.6 \%)$ & $66(61.7 \%)$ & $13(12.2 \%)$ & $22(20.5 \%)$ & \\
\hline & Class IV & $7(3.3 \%)$ & $152(72.4 \%)$ & $18(8.6 \%)$ & $33(15.7 \%)$ & \\
\hline
\end{tabular}

TABLE 7: Awareness about the purpose of condom use (n-724)

\begin{tabular}{|c|c|c|c|c|c|c|}
\hline Category & & Correct & $\begin{array}{l}\text { Partially } \\
\text { correct }\end{array}$ & Wrong & Don't know & Chi-square test \\
\hline \multirow[b]{2}{*}{ Sex } & Male & $49(24.3 \%)$ & $78(18.2 \%)$ & $15(12.4 \%)$ & $60(44.5 \%)$ & \multirow{2}{*}{$\begin{array}{l}\mathrm{x}^{2}-41.6 ; \mathrm{p} \\
\text { value < } \\
0.01 ; \text { Significant } \\
\text { Association }\end{array}$} \\
\hline & Female & $68(13 \%)$ & $123(31.2 \%)$ & $54(8.4 \%)$ & $277(47.3 \%)$ & \\
\hline \multirow{5}{*}{$\begin{array}{l}\text { Age } \\
\text { Groups }\end{array}$} & $<20$ years & $21(8.1 \%)$ & $55(21.3 \%)$ & $40(15.5 \%)$ & $142(55.1 \%)$ & \multirow{5}{*}{$\begin{array}{l}\mathrm{x}^{2}-55.8 ; \mathrm{p}- \\
\text { value }<0.01 ; \\
\text { Significant } \\
\text { Association }\end{array}$} \\
\hline & $20-29$ years & $27(17.7 \%)$ & $39(25.5 \%)$ & $14(9.1 \%)$ & $73(47.7 \%)$ & \\
\hline & $30-39$ years & $46(22.6 \%)$ & $71(34.8 \%)$ & $9(4.4 \%)$ & $78(38.2 \%)$ & \\
\hline & $40-49$ years & $18(24.7 \%)$ & $26(35.6 \%)$ & $2(2.7 \%)$ & $27(37 \%)$ & \\
\hline & $50 \&$ above & $5(13.9 \%)$ & $10(27.8 \%)$ & $4(11.1 \%)$ & $17(47.2 \%)$ & \\
\hline \multirow{5}{*}{ Education } & Illiterate & $1(2.4 \%)$ & $5(12.2 \%)$ & $10(24.4 \%)$ & $25(61 \%)$ & \multirow{5}{*}{$\begin{array}{l}\mathrm{x}^{2}-76.6 ; \mathrm{p} \\
\text { value }<0.01 ; \\
\text { Significant } \\
\text { Association }\end{array}$} \\
\hline & Primary & $11(9.8 \%)$ & $21(18.8 \%)$ & $22(19.6 \%)$ & $58(51.8 \%)$ & \\
\hline & Secondary & $73(15.6 \%)$ & $136(29.1 \%)$ & $30(6.4 \%)$ & $229(48.9 \%)$ & \\
\hline & $\begin{array}{l}\text { Higher } \\
\text { Secondary }\end{array}$ & $28(29.8 \%)$ & $35(37.2 \%)$ & $6(6.4 \%)$ & $25(26.6 \%)$ & \\
\hline & $\begin{array}{l}\text { Graduate \& } \\
\text { above }\end{array}$ & $4(44.4 \%)$ & $4(44.4 \%)$ & $1(11.1 \%)$ & $0(0 \%)$ & \\
\hline \multirow{4}{*}{ Occupation } & Self employed & $9(31 \%)$ & $13(44.8 \%)$ & $1(3.5 \%)$ & $6(20.7 \%)$ & \multirow{4}{*}{$\begin{array}{l}\mathrm{x}^{2}-36.1 ; \mathrm{p}- \\
\text { value }<0.01 \\
\text { Significant } \\
\text { Association }\end{array}$} \\
\hline & $\begin{array}{l}\text { Public-private } \\
\text { sector employee }\end{array}$ & $13(22 \%)$ & $21(35.6 \%)$ & $4(6.8 \%)$ & $21(35.6 \%)$ & \\
\hline & $\begin{array}{l}\text { Unskilled } \\
\text { laborer }\end{array}$ & $69(18.5 \%)$ & $109(29.1 \%)$ & $30(8)$ & $166(44.4 \%)$ & \\
\hline & Unemployed & $26(9.9 \%)$ & $58(22.1 \%)$ & $34(13 \%)$ & $144(55 \%)$ & \\
\hline \multirow{3}{*}{ Religion } & Hindu & $115(16.2 \%)$ & $198(27.9 \%)$ & $67(9.4 \%)$ & $330(46.5 \%)$ & \multirow{3}{*}{$\begin{array}{l}\mathrm{x}^{2}-1 ; \mathrm{p} \text { value } \\
<0.9 ; \text { Non- } \\
\text { Significant } \\
\text { Association }\end{array}$} \\
\hline & Muslim & $1(14.3 \%)$ & $1(14.3 \%)$ & $1(14.3 \%)$ & $4(57.1 \%)$ & \\
\hline & Others & $1(14.3 \%)$ & $2(28.6 \%)$ & $1(14.3 \%)$ & $3(42.8 \%)$ & \\
\hline \multirow[b]{2}{*}{ Caste } & Backward Class & $39(13.3 \%)$ & $69(23.5 \%)$ & $33(11.2 \%)$ & $153(52 \%)$ & \multirow{2}{*}{$\begin{array}{l}\mathrm{x}^{2}-10.1 ; \mathrm{p} \\
\text { value }< \\
0.01 ; \text { Significant } \\
\text { Association }\end{array}$} \\
\hline & Others & $78(18.1 \%)$ & $132(30.7 \%)$ & $36(8.4 \%)$ & $184(42.8 \%)$ & \\
\hline \multirow{3}{*}{$\begin{array}{l}\text { Marital } \\
\text { Status }\end{array}$} & Married & $81(19.9 \%)$ & $130(31.9 \%)$ & $24(5.9 \%)$ & $173(42.4 \%)$ & \multirow{3}{*}{$\begin{array}{l}\mathrm{x}^{2}-32 ; \mathrm{p} \text {-value } \\
<0.01 ; \\
\text { Significant } \\
\text { Association }\end{array}$} \\
\hline & Unmarried & $33(11.1 \%)$ & $66(22.2 \%)$ & $44(14.8 \%)$ & $155(52 \%)$ & \\
\hline & Divorced \& & $3(16.7 \%)$ & $5(27.8 \%)$ & $1(5.6 \%)$ & $9(50 \%)$ & \\
\hline
\end{tabular}


Assessment Of Knowledge And Attitude Of Rural Population About HIV/AIDS In Raigad

\begin{tabular}{|l|l|l|l|l|l|l|}
\hline & others & & & & & \\
\hline \multirow{2}{*}{\begin{tabular}{l} 
Per capita \\
\multirow{2}{*}{$\begin{array}{l}\text { mome per } \\
\text { month }\end{array}$}
\end{tabular}} & Class I & $12(41.4 \%)$ & $12(41.4 \%)$ & $2(6.9 \%)$ & $3(10.3 \%)$ & $\mathrm{x}^{2}-35.8 ; \mathrm{p}$ \\
\cline { 2 - 7 } & Class II & $14(25 \%)$ & $19(33.9 \%)$ & $4(7.2 \%)$ & $19(33.9 \%)$ & value $<0.01 ;$ \\
\cline { 2 - 6 } & Class III & $33(18.2 \%)$ & $53(29.3 \%)$ & $14(7.7 \%)$ & $81(44.8 \%)$ & Significant \\
\cline { 2 - 6 } & Class IV & $58(12.7 \%)$ & $117(25.6 \%)$ & $49(10.7 \%)$ & $234(51.1 \%)$ & Association \\
\hline
\end{tabular}

TABLE 8: Composite score of knowledge(n -724)

\begin{tabular}{|c|c|c|c|c|}
\hline Questions & $\begin{array}{c}\text { Max } \\
\text { marks }\end{array}$ & Score & Average score & $\%$ \\
\hline Heard of STI? & 1 & 327 & 0.45 & 45.17 \\
\hline Are STIs curable? & 1 & 87 & 0.12 & 12.02 \\
\hline Have you heard of HIV/AIDS? & 1 & 626 & 0.86 & 86.46 \\
\hline $\begin{array}{l}\text { Is there any difference between HIV } \\
\& \text { AIDS? }\end{array}$ & 1 & 178 & 0.25 & 24.59 \\
\hline $\begin{array}{l}\text { Is there any relation between } \\
\text { HIV/AIDS \& STIs? }\end{array}$ & 1 & 198 & 0.27 & 27.35 \\
\hline Is HIV/AIDs completely curable? & 1 & 414 & 0.57 & 57.18 \\
\hline Availability of HIV testing facilities? & 1 & 195 & 0.27 & 26.93 \\
\hline $\begin{array}{l}\text { Availability of HIV treatment } \\
\text { facilities? }\end{array}$ & 1 & 222 & 0.31 & 30.66 \\
\hline Desire more information on HIV? & 1 & 637 & 0.88 & 87.98 \\
\hline Have you heard of condom? & 1 & 397 & 0.55 & 54.83 \\
\hline Do you discuss health and sex issues? & 1 & 404 & 0.56 & 55.80 \\
\hline $\begin{array}{l}\text { Necessity of HIV testing in pregnant } \\
\text { women? }\end{array}$ & 1 & 325 & 0.45 & 44.89 \\
\hline Undergone HIV testing? & 1 & 95 & 0.13 & 13.12 \\
\hline Meaning of STIs? & 2 & 110 & 0.15 & 15.19 \\
\hline Ways of transmission of STIs? & 2 & 166 & 0.23 & 22.93 \\
\hline Preventive measures for STIs? & 2 & 160 & 0.22 & 22.10 \\
\hline Symptoms of HIV? & 2 & 208 & 0.29 & 28.73 \\
\hline Symptoms of AIDS? & 2 & 248 & 0.34 & 34.25 \\
\hline Transmission of HIV/AIDS? & 2 & 443 & 0.61 & 61.19 \\
\hline Preventive measures for HIV/AIDS? & 2 & 319 & 0.44 & 44.06 \\
\hline Purpose of condom use? & 2 & 435 & 0.60 & 60.08 \\
\hline Total & 29 & 6194 & 0.30 & 29.50 \\
\hline
\end{tabular}

TABLE 9: Composite score of attitude (n-724)

\begin{tabular}{|c|c|c|c|}
\hline Statements & Score & Average score & Percent \\
\hline $\begin{array}{l}\text { STIs can be cured by having sex relation with young } \\
\text { unmarried woman? }\end{array}$ & 542 & 0.75 & 74.86 \\
\hline $\begin{array}{l}\text { It is mandatory to subjects both the partners for HIV } \\
\text { testing before marriage? }\end{array}$ & 28 & 0.04 & 3.87 \\
\hline $\begin{array}{l}\text { It is dangerous to keep HIV infected individual at } \\
\text { home? }\end{array}$ & 304 & 0.42 & 41.99 \\
\hline $\begin{array}{l}\text { Complete elimination of prostitution is necessary to } \\
\text { prevent HIV transmission? }\end{array}$ & 81 & 0.11 & 11.19 \\
\hline HIV infected females should never get conceived? & 269 & 0.37 & 37.15 \\
\hline $\begin{array}{l}\text { Those who are confirmed HIV negative after testing, } \\
\text { have no risk of becoming HIV infected any time in the } \\
\text { life? }\end{array}$ & 384 & 0.53 & 53.04 \\
\hline It is possible to identify HIV infected individual easily? & 391 & 0.54 & 54.01 \\
\hline
\end{tabular}


Assessment Of Knowledge And Attitude Of Rural Population About HIV/AIDS In Raigad

\begin{tabular}{|l|c|c|c|}
\hline STIs, \& HIV/AIDS are transmitted by only females ? & 427 & 0.59 & 58.98 \\
\hline It is necessary to test each individual for HIV infection? & 31 & 0.04 & 4.28 \\
\hline $\begin{array}{l}\text { It is dangerous to provide health care to HIV infected } \\
\text { individual? }\end{array}$ & 306 & 0.42 & 42.27 \\
\hline \multicolumn{1}{|c|}{ Total } & 2763 & 0.38 & 38.16 \\
\hline
\end{tabular}

TABLE 10: Association of average score of attitude and knowledge with awareness of HIV/AIDS (n-724)

\begin{tabular}{|c|c|c|c|c|c|c|c|}
\hline \multirow{2}{*}{\multicolumn{2}{|c|}{ Category }} & \multicolumn{2}{|c|}{ Knowledge } & \multirow{2}{*}{$\begin{array}{c}\text { Chi-square } \\
\text { test }\end{array}$} & \multicolumn{2}{|c|}{ Attitude } & \multirow{2}{*}{$\begin{array}{c}\text { Chi- } \\
\text { square } \\
\text { test }\end{array}$} \\
\hline & & $\operatorname{Poor}(\mathbf{0}-13)$ & Good(14-29) & & $\underline{\operatorname{Poor}(0-5)}$ & Good(6-10) & \\
\hline \multirow[b]{2}{*}{ Sex } & Male & 155 & 47 & \multirow[b]{2}{*}{$\begin{array}{l}\mathrm{P}<0.01 ; \\
\text { Significant }\end{array}$} & 146 & 56 & \multirow{2}{*}{$\begin{array}{l}\mathrm{P}- \\
0.049 ; \\
\text { significa } \\
\text { nt }\end{array}$} \\
\hline & Female & 463 & 59 & & 413 & 109 & \\
\hline \multirow{5}{*}{$\begin{array}{l}\text { Age } \\
\text { group } \\
\text { in years }\end{array}$} & $<20$ & 232 & 26 & \multirow{5}{*}{$\begin{array}{l}\text { P- } 0.09 ; \\
\text { Non- } \\
\text { significant }\end{array}$} & 220 & 38 & \multirow{5}{*}{$\begin{array}{l}\mathrm{P}< \\
0.01 \text {; } \\
\text { significa } \\
\text { nt }\end{array}$} \\
\hline & $20-29$ & 130 & 23 & & 118 & 35 & \\
\hline & $30-39$ & 168 & 36 & & 146 & 58 & \\
\hline & $40-49$ & 58 & 15 & & 47 & 26 & \\
\hline & $50 \&$ above & 30 & 6 & & 28 & 8 & \\
\hline \multirow{5}{*}{$\begin{array}{l}\text { Educati } \\
\text { on }\end{array}$} & Illiterate & 41 & 0 & \multirow{5}{*}{$\begin{array}{l}\mathrm{P}<0.01 \\
\text { Significant }\end{array}$} & 37 & 4 & \multirow{5}{*}{$\begin{array}{l}\mathrm{P}< \\
0.01 \text {; } \\
\text { significa } \\
\text { nt }\end{array}$} \\
\hline & Primary & 109 & 3 & & 94 & 18 & \\
\hline & Secondary & 413 & 55 & & 371 & 97 & \\
\hline & $\begin{array}{l}\text { Higher } \\
\text { Secondary }\end{array}$ & 55 & 39 & & 57 & 37 & \\
\hline & $\begin{array}{l}\text { Graduate \& } \\
\text { above }\end{array}$ & 0 & 9 & & 0 & 9 & \\
\hline \multirow{4}{*}{$\begin{array}{l}\text { Occupa } \\
\text { tion }\end{array}$} & Self employed & 12 & 17 & \multirow{4}{*}{$\begin{array}{l}\mathrm{P}<0.01 ; \\
\text { significant }\end{array}$} & 16 & 13 & \multirow{4}{*}{$\begin{array}{l}\mathrm{P}< \\
0.01 \text {; } \\
\text { significa } \\
\text { nt }\end{array}$} \\
\hline & $\begin{array}{l}\text { Public-private } \\
\text { sector } \\
\text { employee }\end{array}$ & 43 & 16 & & 40 & 19 & \\
\hline & $\begin{array}{l}\text { Unskilled } \\
\text { laborer }\end{array}$ & 318 & 56 & & 295 & 79 & \\
\hline & Unemployed & 245 & 17 & & 208 & 54 & \\
\hline \multirow{3}{*}{$\begin{array}{l}\text { Religio } \\
\mathrm{n}\end{array}$} & Hindu & 607 & 103 & \multirow{3}{*}{$\begin{array}{l}\mathrm{P}-1.1 ; \\
\text { Non- } \\
\text { significant }\end{array}$} & 549 & 161 & \multirow{3}{*}{$\begin{array}{l}\mathrm{P}-0.8 ; \\
\text { non- } \\
\text { significa } \\
\text { nt }\end{array}$} \\
\hline & Muslim & 6 & 1 & & 5 & 2 & \\
\hline & Others & 5 & 2 & & 5 & 2 & \\
\hline \multirow{2}{*}{ Caste } & $\begin{array}{l}\text { Backward } \\
\text { Class }\end{array}$ & 255 & 39 & \multirow{2}{*}{$\begin{array}{l}\mathrm{P}-0.4 \\
\text { Non- } \\
\text { significant }\end{array}$} & 235 & 59 & \multirow{2}{*}{$\begin{array}{l}\mathrm{p}-2.1 ; \\
\text { non- } \\
\text { significa } \\
\mathrm{nt}\end{array}$} \\
\hline & Others & 363 & 67 & & 324 & 106 & \\
\hline \multirow{3}{*}{$\begin{array}{l}\text { Marital } \\
\text { Status }\end{array}$} & Married & 338 & 70 & \multirow{3}{*}{$\begin{array}{l}\text { p- } 0.07 ; \\
\text { Non- } \\
\text { significant }\end{array}$} & 304 & 104 & \multirow{3}{*}{$\begin{array}{l}\mathrm{p}-0.14 ; \\
\text { Non- } \\
\text { significa } \\
\text { nt }\end{array}$} \\
\hline & Unmarried & 265 & 33 & & 241 & 57 & \\
\hline & $\begin{array}{l}\text { Divorced \& } \\
\text { others }\end{array}$ & 15 & 3 & & 14 & 4 & \\
\hline \multirow{4}{*}{$\begin{array}{l}\text { Per } \\
\text { capita } \\
\text { income } \\
\text { per } \\
\text { month }\end{array}$} & Class I & 14 & 15 & \multirow{4}{*}{$\begin{array}{c}\mathrm{P}<0.01 ; \\
\text { Significant }\end{array}$} & 18 & 11 & \multirow{4}{*}{$\begin{array}{l}\mathrm{P}-0.23 \\
\text { non- } \\
\text { significa } \\
\text { nt }\end{array}$} \\
\hline & Class II & 39 & 17 & & 42 & 14 & \\
\hline & Class III & 159 & 22 & & 140 & 41 & \\
\hline & Class IV & 406 & 52 & & 359 & 99 & \\
\hline
\end{tabular}

IV. Disscussion

A community based descriptive epidemiological study was carried out in 40 villages adopted by PRIDE-India NGO in Mahad Taluka of Raigad District. The study period was January 2009 to April 2009.

Total 724 individuals was interviewed with 202 (27.9\%) males and $522(72.1 \%)$ females. Most of them $615(84.94 \%)$ were in the age group of 15 - 40 years, Hindu by religion $(710,98.06 \%)$, backward class $(294$, 
Assessment Of Knowledge And Attitude Of Rural Population About HIV/AIDS In Raigad

$40.60 \%)$, married $(408,56.35 \%)$, educated up to secondary school level $(480,66.29 \%)$, occupied as unskilled labourer $(374 / 479,78.8 \%)$ and socio-economic class IV $(458,63.26 \%)$. Also, $547(75.55 \%)$ of participants have also reported various forms of addictions among their family members mainly tobacco consumption (504, $92.13 \%)$ in various forms. Only $99(49.0 \%)$ males and $83(15.90 \%)$ females mentioned that they were the decision makers on their health problems, while, only 87 (43.07\%) males and $288(55.17 \%)$ females said that they discuss health and sexual issues with family members.

In the present study, $626(86.46 \%)$ participants had heard of HIV / AIDS. In National Behavioural Surveillance Survey, 2006 carried out by NACO, it was observed that percentage of respondents who had ever heard of HIV / AIDS in rural Maharashtra was $94.5 \%{ }^{\left({ }^{(6)}\right.}$ The results from BBC World Service Trust's HIV knowledge attitudes and practices in India, baseline survey done in 2005 with help of World Bank showed that $82 \%$ of the rural Indian population had heard of AIDS and $64 \%$ had heard of HIV, while $63 \%$ had heard of both HIV and AIDS. ${ }^{(7)}$

In this study level of awareness was significantly lower in females, individuals aged less than 20 years, illiterate, unemployed backward caste and unmarried population. Level of awareness about HIV / AIDS in males was $96.04 \%$ and in females $82.76 \%$. Low level of awareness among females may be attributed to the fact that rural women have a barrier of shyness between them and the information providers and also they have less access to information and media in a male dominated society. The social values and customs also restrain the people from knowing and discussing about HIV/AIDS since it is mainly Sexually Transmitted Disease (STD).

In National Family Health Survey 3, 2005-06, coordinated by the International Institute for Population Sciences (IIPS) under the aegis of the Government of India, it was observed that $92.5 \%$ males and $81.6 \%$ females in Maharashtra had heard of HIV / AIDS. ${ }^{(8)}$

In National Behavioural Surveillance Survey, 2006 carried out by NACO, it was observed that percentage of respondents who had ever heard of HIV / AIDS in rural Maharashtra was $96.5 \%$ in males and $92.4 \%$ in females. ${ }^{(6)}$ In a study done by T. Subramanian, et al. in Tamil Nadu, it was seen that only $72 \%$ rural women had heard of AIDS ${ }^{(9)}$ Similarly in a study done by D. Balk, S. Lahiri in 13 states of India, low rates of knowledge and awareness were reported more among rural women. ${ }^{(\mathbf{1 0})}$ In another study done by Tillich M. and Nilsson A. amongst pregnant women of rural Maharashtra, in 2002, it was observed that only $81 \%$ rural women had ever heard of HIV / AIDS. ${ }^{(11)}$

In the present study Level of awareness was significantly lower in individuals younger than 20 years (200, 77.52\%) as compared to those older than 20 years $(426,91.42 \%)$. This may be attributed to the fact that adolescent boys and girls are less open with the adults to talk about the issue of HIV / AIDS, and this might be the reason behind some of them avoiding to answer the questions about HIV / AIDS. Similarly, in another study done by D. Balk, S. Lahiri in 13 states of India it was seen that AIDS knowledge was more among those older than 20 years. ${ }^{(12)}$ In National Behavioural Surveillance Survey, 2006 carried out by NACO, level of awareness of HIV / AIDS in age group of 15 to 24 years was found to be $88.5 \%$ and of 25 to 39 years was $84.7 \%$. ${ }^{(6)}$

In the present study level of awareness was significantly lower in illiterate $(21,51.22 \%)$ as compared to literates $(683,88.58 \%)$. As the education level increases the awareness about HIV / AIDS increases, which is because of the fact that illiterate people are less exposed to informative material than educated and the level of understanding of the information increases with education. Similarly in a study conducted on AIDS awareness and knowledge of Indian women, done by D. Balk, S. Lahiri in 13 states of India, low rates of knowledge and awareness were reported more among rural and illiterate women. Multivariate analysis found rural, poorly educated and poor women to be the least likely to be AIDS-aware and, if aware, to be having the poorest understanding of AIDS. ${ }^{(\mathbf{1 0})}$ In National Behavioural Surveillance Survey, 2006 carried out by NACO, it was seen that level of awareness about HIV / AIDS was lower in illiterates (45.8\%) as compared to primary (77.7\%), middle $(91.6 \%)$, secondary and higher secondary $(98.2 \%)$ and graduate and above $(99.8 \%){ }^{(6)}$

In the present study level of awareness was significantly lower in unmarried $(238,79.87 \%)$ as compared to married $(373,91.42 \%)$ and divorced $(15,83.33 \%)$. This may be attributed to the fact that most of the individuals in this study who are unmarried are adolescents and young adults, who are less open with adults to talk about HIV / AIDS and thus may have avoided answering to the questions about HIV / AIDS. In National Behavioural Surveillance Survey, 2006 carried out by NACO, it was seen that the level of awareness of HIV / AIDS in currently married $81.4 \%$, unmarried $92.3 \%$ and formerly married $71.4 \% .^{(6)}$

In this study, mass media i.e. television, radio, etc was major source of information of HIV / AIDS, as it contributed for 316 (74.18\%) of participants, while, peer group i.e. friends and colleagues contributed for 198 (46.48\%) of participants and hospitals and doctors contributed for only $51(11.97 \%)$ of participants. In a study done by D. Balk, S. Lahiri in 13 states of India, it was seen that AIDS knowledge was more among those who had greater exposure to mass media. Television was the greatest source of AIDS information. ${ }^{(\mathbf{1 2})}$ Similarly in another study done by T. Subramanian et al.in Tamil Nadu, television, radio and friends were reported as the main sources of information on AIDS. ${ }^{(9)}$ In National Behavioural Surveillance Survey, 2006 carried out by $\mathrm{NACO}$, it was seen that mass media i.e. television, radio, etc was major source of information (86.6\%) about 
HIV / AIDS in Maharashtra. ${ }^{(6)}$ In another study done by V. Bhatia et al. in Chandigarh, it was seen that major source of information about AIDS was mass media followed by friends. ${ }^{(13)}$

In the present study 285 (39.36\%) individuals were aware about HIV treatment, 195 (26.93\%) individuals were aware about availability of HIV testing facilities and $172(23.76 \%)$ individuals were aware about availability of treatment facilities. This study indicated that participants were willing to gain more knowledge, as 637 (87.98\%) said that they desire more information about HIV / AIDS. Most of the participants were not aware about sexually transmitted infections other than HIV / AIDS (327, 45.16\%).

In the present study most of the individuals were not aware of difference between HIV and AIDS, as only 178 (24.59\%) knew correct difference between HIV and AIDS. There was lot of confusion between understanding of terms HIV and AIDS. Participants were also not able to distinguish between symptoms of HIV and AIDS. This can be attributed to low levels of access and exposure to information on HIV / AIDS as well as shyness to discuss this issue with anyone, as majority of the participants of the present study are females. In a study done by Tillich M. and Nilsson A. amongst pregnant women of rural Maharashtra, in 2002, it was observed that rural women were not able to distinguish between HIV and AIDS. ${ }^{(11)}$ Similar were the findings in another study done by Andersson C et al. among teenagers in Solapur district of Maharashtra, in 2004, where only $25 \%$ of the students in the village were aware of difference between HIV and AIDS. ${ }^{(14)}$

In the present study most of the individuals were not aware of relation between HIV / AIDS and STIs, as only $198(27.35 \%)$ knew correct relation. This can be attributed to low levels of access and exposure to information on HIV / AIDS as well as low level of awareness about different STIs. In the present study only 327 (45.17\%) had ever heard of STIs and very less individuals knew correct $(3,0.41 \%)$ and partially correct $(104$, $14.36 \%)$ meaning of STIs.

In the present study most of the individuals were not aware about correct symptoms of HIV/AIDS, as only $14(1.93 \%)$ participants had correct knowledge and 220 (30.39\%) had partially correct knowledge about symptoms of AIDS. This can be attributed to low levels of access and exposure to information on HIV / AIDS as well as shyness to discuss this issue with anyone, as majority of the participants of the present study are females. Twenty different symptoms were mentioned for AIDS, which included fever (198, 27.35\%), weight loss $(201,27.76 \%)$, weakness $(165,22.79 \%)$, cough $(129,17.82 \%)$, diarrhoea $(108,14.92 \%)$, Tuberculosis $(104$, $14.36)$, decreased appetite $(87,12.02 \%)$, cachexia $(65,8.98 \%)$, cancer $(64,8.84 \%)$, vomiting $(51,7.18 \%)$, Herpes rash $(49,6.77 \%)$, malaise $(42,5.80 \%)$, blindness $(41,5.66 \%)$, giddiness $(35,4.83 \%)$, fungal infections $(32,4.42 \%)$, per vaginal white discharge $(29,4.01 \%)$ and a few weird responses, like worms $(22,3.04 \%)$, blackening of skin $(19,2.62 \%)$, hair fall $(1.38 \%)$, increased libido $(8,1.10 \%)$. Similarly in a study done by Subramanian $\mathrm{T}$ et al. in Tamil Nadu, it was observed that when asked symptoms of AIDS, $28 \%$ mentioned of fever and $39 \%$ about weight loss and correct responses were more from literates. ${ }^{(9)}$

In the present study most of the individuals were not aware about correct ways of transmission of HIV / AIDS, as only $60(8.29 \%)$ participants had correct knowledge and $323(44.61 \%)$ had partially correct knowledge about ways of transmission of HIV / AIDS. This can be attributed to low levels of access and exposure to information on HIV / AIDS as well as the social values and customs which restrain the people from knowing and discussing about HIV/AIDS.

In this study, level of awareness about transmission of HIV was significantly lower in illiterates (10, $24.39 \%)$ as compared to literates $(373,54.61 \%)$. As the education level increases the awareness about HIV / AIDS increases, which is because of the fact that illiterate people are less exposed to informative material than educated and the level of understanding of the information increases with education. In another study done by Subramanian T et al. in Tamil Nadu, it was seen that there was no significant difference in awareness about HIV transmission among the literates and illiterates $(\mathrm{P}>0.05)$. While $13.2 \%$ of the illiterates and $26.9 \%$ of the literates said 'through infected blood,' 'infected mother to child' was reported by $0.6 \%$ in total, who were all literates. Correct responses were more from literates. ${ }^{(9)}$

In the present study, HIV/AIDS transmits by sexual relations was known to only 292 (44.33\%) individuals, while HIV transmits by infected blood and infected needles was known to 135 (18.65\%) and 111 (15.33\%) individuals respectively. Mother to child transmission of HIV was known to only $144(19.89 \%)$ and only $103(14.23 \%)$ say that it is necessary to HIV testing of all pregnant females. In National Behavioural Surveillance Survey, 2006 done by National AIDS Control Organization, it was observed that in rural Maharashtra, $86.8 \%$ males and $83.1 \%$ females knew that HIV can be transmitted through sexual contacts, $91.5 \%$ males and $81.1 \%$ females knew infected blood transfusion, $86.0 \%$ males and $80.5 \%$ females knew about infected needle sharing and $75.1 \%$ males and $73.6 \%$ females knew about mother to child transmission of HIV. ${ }^{(6)}$ In another study done by Subramanian $\mathrm{T}$ et al. in Tamil Nadu, 52.5\% of the women mentioned that spread of HIV was through unprotected sexual contact. ${ }^{(9)}$

The present study brought up many misconceptions of people about HIV transmission, as a few individuals even mentioned mosquito bite $(29,4.01 \%)$, kissing $(27,3.73 \%)$, sharing food $(22,3.04 \%)$ and clothes $(16,2.21 \%)$, coughing $(11,1.52 \%)$, through urine $(8,1.10 \%)$, through water $(2,0.28 \%)$, attending 
funeral of person who died due to AIDS $(2,0.28 \%)$, familial or hereditary $(2,0.28 \%)$ as ways of transmission of HIV / AIDS. Though the percentages of the misconceptions were low, these issues need to be addressed as they increase the fear and stigma of HIV / AIDS in the community. Many times people taking care of HIV infected persons are also stigmatised. In many villages the HIV infected persons as well as their families are out casted. All this results in neglect of the HIV infected individuals and increase in social difference. In National Behavioural Surveillance Survey, 2006 it was observed that in rural Maharashtra, 23.4\% males and 23.9\% females mentioned mosquito bite as mode of transmission of HIV. ${ }^{(6)}$

In another study done by Andersson C et al. among teenagers in Solapur district of Maharashtra, in 2004, it was observed that, $36.9 \%$ of the students thought that kissing is one of the main ways that HIV is spread by. $7.1 \%$ of the female students and $2.1 \%$ of the male students in the village felt HIV is spread when eating or drinking from the same plates and cups. ${ }^{(\mathbf{1 4})}$

In the present study most of the individuals were not aware about correct measures of prevention of HIV / AIDS, as only 30 (4.14\%) participants had correct knowledge and 259 (35.77\%) had partially correct knowledge about measures of prevention of HIV / AIDS. This can be attributed to low levels of access and exposure to information on HIV / AIDS as well as the social values and customs which restrain the people from knowing and discussing about HIV/AIDS. This study indicated that, level of awareness (correct plus partially correct responses) was significantly lower in individuals aged less than 20 years $(57,22.09 \%)$, illiterate $(6$, $14.63 \%)$, unemployed $(91,34.73 \%)$ and socio-economic class IV $(159,34.72 \%)$. HIV/AIDS can be prevented by using condom every time was known to only 233 (32.18\%) individuals, while abstinence from sex was mentioned by 154 (21.27\%), while using new needles / syringes was known to only 122 (16.85\%), using HIV tested blood was known to 98 (13.54\%) individuals and only 103 (14.23\%) say that it is necessary to do HIV testing of all pregnant females. In another study done by Subramanian $\mathrm{T}$ et al. in Tamil Nadu, in rural India it was observed that, $45 \%$ mentioned of avoiding unprotected sexual contacts; nearly one-fifth of the women reported of the use of disposable/ sterilized needles and syringes. In this aspect, correct response was more $(24.2 \%)$ from literates. In all, use of condom was mentioned by only $2.5 \%$ of the women. ${ }^{(9)}$

Many misconceptions of people about HIV prevention were seen during this study, as few individuals even mentioned mosquito nets $(22,3.04 \%)$, avoiding people who are HIV positive $(38,5.25 \%)$, not sharing food (19, $2.62 \%)$ and clothes $(11,1.52 \%)$, do not attend funeral of person who died due to AIDS $(2,0.28 \%)$ and use of clean drinking water $(2,0.28 \%)$ as ways of prevention of HIV / AIDS.

The present study reveal that even those people who knew about ways of transmission of HIV, were less aware about preventive measures for HIV, as 30 (7.83\%) had correct knowledge and $259(67.62 \%)$ had partially correct knowledge about preventive measures for HIV. This may be partly due to lack of ability or motivation among individuals to improve their awareness status from 'know about transmission' to 'know about prevention' and also due to lack of facilities and resources to improve their knowledge and skills.

Only 397 (54.83\%) were aware about condoms in this study. This may be attributed to low level of exposure to information about condoms and shyness to talk about this issue, as majority of the participants were females. Many of the participants avoided answering questions about condoms. In this study level of awareness about condoms was significantly lower in females $(219,41.95 \%)$ as compared to males $178(88.12 \%)$. This may be due to shyness of rural women to talk openly about issue of condoms as well as lack of facilities and resources to gain knowledge about the same in the rural community. It was also observed that, mass media i.e. television and radio $(278,70.02 \%)$ and peer group i.e. friends and colleagues $(183,46.10 \%)$ were the major sources of information about condom.

In the present study it was observed that many people are not aware about purpose of condom use correctly. $117(16.16 \%)$ had correct knowledge and 201 (27.76\%) had partially correct knowledge about purpose of condom use. This may be due to lack of exposure to information about condoms as well as shyness amongst participants. In a study done by Sunder Lal et al. on Evaluation of Intensive Information, Education and Communication Campaign on HIV/AIDS in rural areas, it was found that effective knowledge of safe sex by use of condom was endorsed by only one third of individuals only. ${ }^{(15)}$ In National Behavioural Surveillance Survey, 2006 it was found that $69.7 \%$ of males and $63.3 \%$ of females in rural Maharashtra knew about purpose of condom use for HIV / AIDS prevention. ${ }^{(6)}$ In national Family Health Survey 3, it was found that $69.4 \%$ males and $31.4 \%$ females in rural Maharashtra knew that consistent condom use can reduce the chances of getting HIV/AIDS. ${ }^{(16)}$ In the present study only $233(32.18 \%)$ individuals mentioned that correct and consistent use of condom can prevent HIV / AIDS, while $123(16.99 \%)$ mentioned prevention of other STIs also. Condom as a contraceptive device was known to $286(39.50 \%)$ individuals. Some $(109,15.06 \%)$ of the participants mentioned use of condom for safe sex. A few $(58,8.01 \%)$ individuals also mentioned use of condom for 'cleanliness of genital organs' and a few $(49,6.77 \%)$ even mentioned use of condom 'to keep extramarital and premarital affairs'.

Maximum obtainable score from knowledge questionnaire was 29, which none of the participants achieved. Maximum score achieved by participant was 24 and minimum score was 2 and average score per 
Assessment Of Knowledge And Attitude Of Rural Population About HIV/AIDS In Raigad

person was 8.56. Composite score for knowledge was only $29.50 \%$. This means, overall knowledge of all the individuals about HIV / AIDS is very poor.

In the present study it was observed that majority $618(85.36 \%)$ individuals had poor score on knowledge scale and only $106(14.64 \%)$ could score more than 13 on knowledge composite score scale.

Maximum obtainable score from attitude questionnaire was 10, which none of the participants achieved. Maximum score achieved by participant was 8 and minimum score was 1 and average score per person was 3.81. Average composite score for attitude was 38.16\%. 165 (22.8\%) individuals had right attitude (Composite score 6 to 10) towards HIV/AIDS. Attitude of the participants towards HIV / AIDS was poor which may be attributed to lack of awareness about the disease.

Composite score for attitude was significantly lower in females, individuals aged less than 20 years, illiterate and unemployed individuals as only $109(20.88 \%)$ females, $38(14.73 \%)$ individuals aged less than 20, $4(9.76 \%)$ illiterate and $54(20.61 \%)$ unemployed individuals could score more than 5 on attitude scale. This reflects poor attitude and lack of acceptability of individuals towards HIV / AIDS. This can be attributed to various factors such as low levels of awareness, lack of access to information, low literacy and various misconceptions about HIV / AIDS.

\section{Conclusion}

It was evident from the study that there was lack of awareness about HIV / AIDS, different STIs as well as condoms, especially among females, adolescents, illiterates, unemployed and poor; indicating need for awareness programmes in these groups.

Thus the knowledge of rural people about HIV / AIDS, its transmission and prevention should be improved with help of vigorous campaigning, training workshops, HIV / AIDS education sessions with special focus on migrant workers and their families.

HIV / AIDS awareness campaigns should be increased at the time of religious festivals and fairs, as migrant workers tend to visit their native place during this period. Proper counselling and testing facilities should be made available to those who are willing for HIV testing.

[1] AIDS epidemic update - December 2009 [Internet]. Geneva Joint United Nations Programme on HIV/AIDS (UNAIDS), World $\begin{array}{lllll}\text { Health } & \text { Organization } & \text { (WHO); } & 2009 & \text { December.6 Available }\end{array}$ http://data.unaids.org/pub/Report/2009/JC1700 Epi Update 2009 en.pdf

[2] HIV/AIDS in India [Internet]. The World Bank; 2009 February [updated 2009 February]. Available from: http://siteresources.worldbank.org/SOUTHASIAEXT/Resources/223546-1192413140459/4281804-1231

[3] Annual Report 2009-10. National AIDS Control Organization, Department of AIDS Control, Ministry of Health and Family Welfare, Government of India; 2009.:4-5.

[4] National AIDS Control Programme. Directorate of Health Services, Public Health Department, Government of Maharashtra. 2010. Available from: http://www.maha-arogya.gov.in/programs/nhp/aids/default.htm

[5] Saggurti N, Verma R K, Jain A, Achyut P, Ramarao S. Patterns and implications of male migration for HIV prevention strategies in Maharashtra, India. Population Council India; 2008 June. Technical brief no. 3:1-3

[6] National Behavioral Surveillance Survey - General population. National AIDS Control Organization, Ministry of Health and Family Welfare, Government of India. 2006:36. Page no. 32, 42-46, 50, 108.

[7] HIV / AIDS knowledge, attitudes and practices in India, Baseline survey, BBC World Service Trust. $2005 ; 15$.

[8] National Family Health Survey (NFHS-3) 2005-06. International Institute for Population Sciences (IIPS) and Macro International. Mumbai: India. 2007; Volume I, Chapter 11:333.

[9] Subramanian T, Gupte MD, Ezhil R. AIDS: An understanding in rural women of South-India. Indian Journal of Sexually Transmitted Diseases. 2007; 28(1):10-14.

[10] Balk D, Lahiri S. Awareness and knowledge of AIDS among Indian women: Evidence from 13 States. Health Transit Rev. 1997; 7:421-65.

[11] Tillich M, Nilsson A. Awareness, attitude and prevention of HIV among pregnant women in Maharashtra State, India. Göteborg, Sweden. The Sahlgrenska Academy at Göteborg University. 2002; :9-15. Available from: www.heartintl.net/HEART/030109/Awareness, Attitude.pdf

[12] Lahiri S, Balk D, Pathak KB. Women in 13 states have little knowledge of AIDS. Natl Fam Health Surv Bull. 1995 Oct; (2):1-4.

[13] Bhatia V, Puri S, Mangat C \& Swami H. An Intervention study to enhance AIDS awareness among underprivileged population in Chandigarh. The Internet Journal of Health [Internet]. 2010; 11 (1). Available at: http://www.ispub.com/journal/the internet journal of health/volume 11 number 1 10/article/an-intervention-study-to-enhanceaids-awareness-among-underprivileged-population-in-chandigarh-1.html

[14] Andersson C, Westergren C. Still scant and insufficient knowledge about HIV/AIDS among teenagers in Solapur District, Maharashtra State, India. Gothenburg, Sweden. The Sahlgrenska Academy at University of Gothenburg. 2004; :8-14. Available at: http://www.vgregion.se/upload/SkaS/FoU/HIVIndia.pdf

[15] Lal S, Vashisht B, Singh M, Punia M S. Evaluation of Intensive Information, Education and Communication Campaign on HIV/AIDS in rural areas. Indian Journal of Community Medicine. 1999; 24(4):175-80.

[16] National Family Health Survey (NFHS-3) 2005-06, Maharashtra fact sheet [Internet]. International Institute for Population Sciences (IIPS) and Macro International. Mumbai, India. 2007. Available from: http://www.nfhsindia.org/pdf/Maharashtra.pdf 